\title{
Interview Dr. Bernhard Doll, Orange Hills
}

\section{Interview Question}

Orange Hills is an expert in Rapid Transformation. Let us imagine the following situation. Company $\mathrm{X}$ has decided to engage in frugal innovation. How can it change its business in this direction?

\section{Author: Dr. Bernhard Doll, Orange Hills}

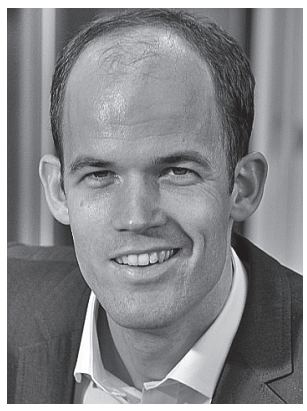

The first question that comes into my mind is: "Why should a company change its business focus to design and deliver "frugal innovation' to customers?" Is it to open up new customer segments to enable a new or "overlooked" population of people to begin owning or using an innovative product or service? Or is the intention more about using the idea of "frugal innovation" to manage risks and complexity inherent in any innovation process by producing "focused" products or services with less means? We see "frugal innovation" as an important concept for companies serving both developing and developed markets, even if the underlying purpose can vary a lot.

Designing and delivering "frugal innovation" requires a certain skill set, which is not quite common in many R\&D departments of companies across Europe. The starting point here is not to wrap technology into a feature-rich product or service that hopefully addresses customers' needs, but to really understand the customer's job a new product or service should try to get done by providing a certain marriage between form and function. Observing the world, understanding contextual constraints, listening to customers to learn and find the right blend of embedded value and reduced waste in the product or service to be designed, is one of the key activities in the "frugal" innovation process.

New tools and methods, and more importantly, a new (human) work culture are required to successfully design "frugal innovation". Social prototyping, ethnography, realtime collaboration, co-creation, crowdsourcing, running projects in 5 weeks rather than 5 months are just few examples of effective methods in this domain. Since "change" is often perceived as peril in larger organizations, however, we have learned in our projects that it is important to define a vision with guiding principles for the new work culture first, but then to start small by running a bunch of rapid innovation projects embodying the principles and facilitated by experienced coaches.

Visual results and "quick wins" lead to visibility within the organization and more and more team members get familiar with the new approach and soak up the work spirit. Later on, the number of people involved can be extended to embrace the entire organization over time.

Bernhard Doll is CEO and co-founder of OrangeHills GmbH, Munich, as well as research fellow at the Center for Leading Innovation \& Cooperation (CLIC) at HHL-Leipzig Graduate School of Management. 